\title{
A MCDM/MADM model for evaluating efficiency of rail-bus transfer
}

\author{
Hanbei Cheng ${ }^{1, a^{*}}$, Zhengdong Huang ${ }^{2, b}$, Xiaojing Hu H,c $^{3, \mathrm{c}}$ \\ ${ }^{1,2,3}$ School of Urban Design, Wuhan University. Donghu Nanlu 8, 430072 Wuhan, China \\ ahanbei.cheng@gmail.com, bhuangwhu@126.com, ‘xjhihappy@163.com
}

Keywords: Transfer; walkability; efficiency; rail station; bus stop

Abstract. Transferability between urban rail and bus transit is an important indicator for evaluating efficiency of a multi-modal transit system. Transit transfer is concerned with distance, safety, comfort, clarity, and equipment applicability. This paper presents a Multi Criteria/Attribute Decision Making (MCDM/MADM) model for measuring transfer efficiency between urban rail station and its feeding bus stops. The model incorporates four criteria and nine indicators for assessing transferability at rail-bus transit nodes. A case study of two typical rail stations in Wuhan city is conducted to illustrate the model. Transferability may be applied for evaluating physical rail-bus connectivity at rail stations, through which transfer problems are identified and improvement plans are suggested.

\section{Introduction}

Public transit systems play a vital role in modern society, especially in China with mass population movement. However, compared to private travel modes, the competitiveness of public transit system is still insufficient ${ }^{[1]}$. The most important reason is the low walkability in rail-bus transfer, caused by lacking integration during infrastructure construction, which is being undervalued in current transportation planning. Besides, the evaluation of pedestrian transfer efficiency, or walkability, between different transit nodes (rail and bus stops) involves loads of relative factors, leading the transferability a multidimensional issue ${ }^{[2]}$.

In this context, literature on factors affecting walking transfer and corresponding methods of measuring performance has dramatically increased in the past two decade. Well-documented evidence demonstrates that the pedestrian transfer efficiency depends largely on two, macro and micro level variables. Marco-level considerations may place an emphasis on unchangeable features(the transfer subjects' social-demographical and travel characteristics), such as income level, age $^{[3]}$, gender, physical conditions ${ }^{[4]}$, disabled or not disabled ${ }^{[5]}$, weather, the frequency and purpose of their walk trip ${ }^{[6]}$. However, micro-level or so-called street-side factors, include walking distance, sidewalk width, adjacent land use and comfort concerns (i.e. crosswalks, street lights or street parking $)^{[7]}$. Targeted on these factors, Monsak Socharoentum ${ }^{[8]}$ introduced the multi-modal transportation with multi-criteria walking (MMT-MCW) to determine optimal walking routes, which involves walking transfer nodes selection technique. David S. Vale ${ }^{[9]}$ combined the node-place model with pedestrian shed ratio to evaluate the pedestrian connectivity of stations areas, integrating land use, transportation and walking conditions based on the original model. Iderlina Mateo-Babiano $^{[6]}$ applied the Analytic Hierarchy Process (AHP) to examine the relative priorities of environmental attributes (alternatives) that satisfy the pedestrian needs, with the end goal of realizing a positive walking environment. Granted, most of researchers used Data Envelopment Analysis (DEA), a non-parametric method introduced by Farrell ${ }^{[10]}$ to measure the relative performance of public transit systems, using multiple criteria as inputs. 


\section{Model}

Micro-level factors influencing transferability among transit modes can be grouped under five criteria, i.e., safety, continuity, comfort, clarity and equipment applicability (Fig. 1). The supplied transferability of a rail station is defined by the average efficiencies of each directions' transferability. For simplicity, this study only considers the first four criteria, in which Continuity is measured by walking time (Eq. 1).

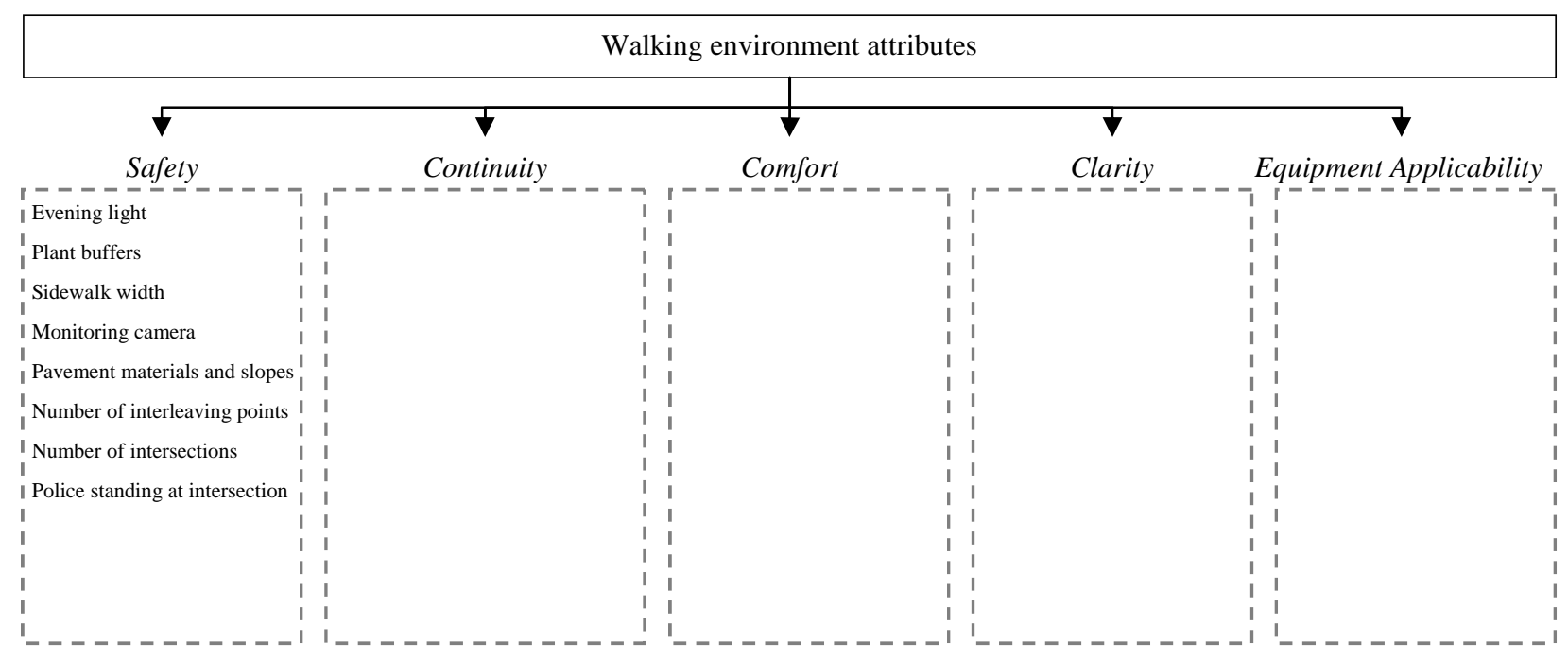

Fig. 1. Interaction between transit travelers and walk environments

$$
C_{T}=\frac{1}{n} C_{i j d}=\frac{1}{n} \sum_{i=1}^{n}\left[x_{\text {time }} \log P W T+x_{\text {safery }}(\log N I P+\log N I+\log P M Q)+x_{\text {continuity }}(\log E D+\log P W D+\log E U D+\log P R D)+x_{\text {clarity }} \log G D F\right]
$$

$C_{T}=$ the general transferability score of rail station $j$

$$
\begin{aligned}
& i=\text { the code of bus stop } \\
& j=\text { the evaluated rail station } \\
& d=\text { the code of entrances of rail station } \\
& n=\text { the number of directions }
\end{aligned}
$$

The evaluation model includes nine indicators, among which three for measuring the safety of the path walking process, four for measuring the continuity, one for measuring the clarity, and one for measuring practical walking time (Fig. 1). The indicators are listed to include walking time (PWT) and distance (PWD), Euclidean distance (EUD), pedestrian route directness ${ }^{[11]}$ (PRD) (the ratio of PWD to EUD), number of intersections (NI), number of interleaving points (NIP), pavement quality (PMQ) (the length ratio of poor segment to the total sidewalk), elevation differences (ED), and direction guiding facilities (DGF) in Eq. (1). In order to keep the normality of the variables, we have log-transformed several indicators, following other reviewed case studies ${ }^{[9]}$. For simplicity, all indicators are assigned equal weights for calculating the final transferability index. It is worth noting that such model does not entail any city or specific stations and as such can be generally applied to any other stations' transfer evaluating.

\section{Application of the model: a case study of Wuhan}

Background. Wuhan is the mainly regional central city situated in the middle part of China with a situation, where the passengers accommodated by the rail system account for $11.2 \%$ in 2013 with an upward trend, while the percentage of routine bus travelling levels off at average $37.4 \%$ from 2010 
onwards. Thus, improving the transfer efficiency between the rail stations and its nearest bus stops will be an effective way to enhance the performance of the whole transit system.

Data collection. In order to fully understand problems in transferability of existing stations, two types of surveys, Pedestrian Survey and Environment Attributes Survey, were conducted in two steps. Due to the collectability of relevant data, two survey were both carried on in off-peak periods (exclusive of 7:00 9:00 and 17:00 19:00). A pilot survey was conducted to check whether the questions are understandable and answerable. The questionnaire was slightly modified after the pilot survey to account for the encountered shortcomings and difficulties.

The Pedestrian Survey, in this case, was conducted to collect two groups' different perceptions of acceptable transferring time and concerns about transfer performance. Consequently, about $97 \%$ of the 31-50 age group was inclined to 5-8 minutes of transferring time, while 18-30 year-old young adults clearly extended the time to 12 minutes. Furthermore, the former were less sensitive to the guiding signs, but more concerned about the sidewalk safety than the young transit travelers. These preferences are treated as the benchmark of evaluating transferring performance.

As to the Environment Attributes Survey, the data of two typical rail stations, Jiedoukou and Baotong Temple, was collected by field survey (Table 1). The elevation difference was figured out by recording the height of each stair and the number of stairs from pedestrian bridge (the highest place in the transferring process) to underground passage or metro platform. As the effectiveness of direction guiding facilities is decided by both people's recognition and perception to the built environment, the factor is measured qualitatively at four hierarchical levels of Good, Fair, Moderate and Poor, scoring at the ascending order between $0-1$ by the transferability cost.

Table 1 The nine indicators of transferring directions and transferability at two rail stations

\begin{tabular}{|c|c|c|c|c|c|c|c|c|c|c|}
\hline & \multirow{3}{*}{$\begin{array}{l}\text { Trans. } \\
\text { score }\end{array}$} & \multicolumn{9}{|c|}{ Criteria and Indicators } \\
\hline & & \multirow{2}{*}{$\begin{array}{l}\text { Time } \\
\text { PWT } \\
\text { [min] }\end{array}$} & \multicolumn{3}{|c|}{ Safety } & \multicolumn{4}{|c|}{ Continuity } & \multirow{2}{*}{$\begin{array}{l}\text { Clarity } \\
\text { DGF }\end{array}$} \\
\hline & & & NIP & $\begin{array}{l}\text { PMQ } \\
{[\%]}\end{array}$ & NI & $\begin{array}{l}\text { ED } \\
{[\mathrm{m}]}\end{array}$ & $\begin{array}{l}\text { PWD } \\
{[\mathrm{m}]}\end{array}$ & $\begin{array}{l}\text { EUD } \\
{[\mathrm{m}]}\end{array}$ & $\begin{array}{l}\text { PRD } \\
{[\%]}\end{array}$ & \\
\hline Origin bus stop & 2.44 & \multicolumn{9}{|c|}{ Destination station: Jiedaokou Rail Station } \\
\hline Luoshi Beiduan-D* & 2.60 & 9.00 & 4 & 0.07 & 3 & 19.55 & 536.59 & 275.02 & 1.95 & 1.00 \\
\hline Jiedaokou Wuluo-D* & 2.63 & 11.00 & 2 & 0.16 & 6 & 19.55 & 819.45 & 766.05 & 1.07 & 0.75 \\
\hline Jiedaokou Wuluo-A* & 2.41 & 6.20 & 0 & 0.15 & 6 & 19.55 & 675.47 & 635.82 & 1.06 & 0 \\
\hline Wugongda-A* & 2.72 & 10.15 & 2 & 0.06 & 4 & 23.45 & 854.83 & 652.89 & 1.31 & 1.00 \\
\hline Wuluo Luoshi-B* & 2.40 & 4.56 & 1 & 0.12 & 3 & 19.55 & 634.22 & 318.76 & 1.99 & 0.75 \\
\hline Jiedaokou Luoyu-C* & 1.97 & 1.00 & 0 & 0.04 & 0 & 19.55 & 322.56 & 260.17 & 1.24 & 0 \\
\hline Luoshi Beiduan-C* & 2.34 & 5.18 & 2 & 0.14 & 1 & 19.55 & 563.59 & 242.21 & 2.33 & 0.75 \\
\hline Origin bus stop & 2.08 & \multicolumn{9}{|c|}{ Destination station: Baotong Temple } \\
\hline Jiedaokou-C1* & 2.28 & 5.36 & 2 & 0.11 & 2 & 14.40 & 356.53 & 328.45 & 1.09 & 0.25 \\
\hline Baotong Temple-B* & 2.10 & 6.17 & 2 & 0.16 & 0 & 14.40 & 312.00 & 243.22 & 1.28 & 0.25 \\
\hline Baotong Temple-A* & 1.48 & 1.00 & 0 & 0 & 0 & 14.40 & 203.77 & 76.14 & 2.67 & 1.00 \\
\hline Dingziqiao-D* & 2.46 & 8.13 & 2 & 0.17 & 4 & 14.40 & 643.27 & 600 & 1.07 & 0.75 \\
\hline
\end{tabular}

*the code of entrance/exit at rail stations

Analysis and interpretation. In this model, data are analyzed to identify the practical problems and transferability of stations as a whole. The specific direction transferring data are analyzed using the Environment Attributes Survey data (Table ) together with criteria and indicators weights to perform the MCDM/MADM model in Eq. 2.

In 错误!未找到引用源。, the transferability score represents the difficulty in transferring process, the greater the value, the lower the efficiency. Evidently, Baotong Temple rail station is 
exhibiting higher transfer efficiency than that of Jiedaokou rail station. Further, in all of the O-D trips, the Jiedaokou Luoyu-C*, the Baotong Temple-A* and Baotong Temple-B* get an overall "good" performance, but the Baotong Temple-A* shows better results with the lowest transferability score than the other two (ranked 2nd and 3rd, respectively). Meanwhile, the Jiedaokou-C1* is ranked the 4 th as it has some deficiencies (except the transferring time), especially in safety concerns due to its surrounding large hospitals and shopping malls. Luoshi Beiduan-C* is ranked 5th with deficiency noted in continuity. Oppositely, the Wugongda- $\mathrm{A}^{*}$ is ranked the last in overall performance, which is mainly attributed to the factor of continuity (Fig. 1). This bus stop is located in the tunnel, transit riders have to climb up the stairs to reach their destination. As revealed in data collection section, the great majority of respondents were inclined to walk 5-8 minutes, which was consistent with 70\% of existing O-D transferring trips, excluding the Luoshi Beiduan-D*, Wugongda-A* and Jiedaokou Wuluo-D*.

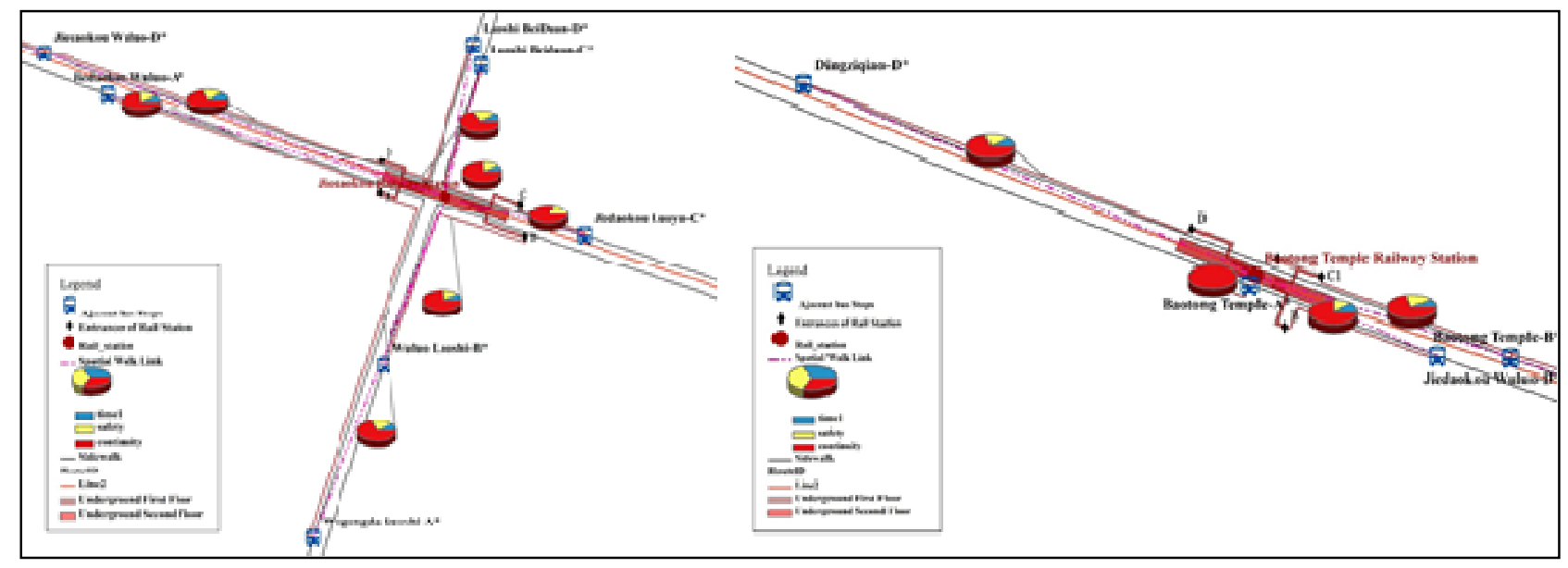

Fig. 2. The transferability of each direction in Jiedaokou (Left) and Baotong Temple (Right) rail station

Recommendations for transfer improvement. Following the analysis of stations, suggestions below are given to enhance the overall transferring performance.

(1) As indicated in Table, approximately $60 \%$ of transferring trips were in the level of moderate quality in direction guiding. Thus, the proper information guiding strategy should be deployed. Detailed guidance information of rail and bus service should be available along the way finding, adjacent shopping malls and underground passage. As Wuhan is going towards internationalization, guidance should be provided with multiple languages.

(2) Safety is another major concern. This includes traffic signals at street crossing, clearance of furniture at entrance to underground rail platform, as well as proper pavement of sidewalks at the ground level.

(3) For most of transferring processes, there are unacceptable performance in pavement quality. Sidewalks are found to be segmented, and are occupied by other uses here and there.

(4) There is an urgent need to re-place bus stops whenever possible, such as the case for Jiedaokou Wuluo, Wugongda and Luoshi Beiduan.

\section{Conclusions and future research}

This paper provides a MCDM/MADM model for evaluating the transfer efficiency of public transit nodes. One of the important features of the model is flexibility and involvement of diverse factors in transit transfer process. As a case study, the proposed model was used to evaluate the existing rail stations and their feeder bus stops in Wuhan. An investigation of two rail stations 
validates the effectiveness of the model. The model results are useful for either comparing rail-bus transferability at different rail stations, or for identifying major factors of built environment existing at an individual rail station. For newly-built rail stations, the model may be applied for evaluating different scenarios of placing stops of feeder buses.

Further research will include three aspects: (1) improving the model with ability to simultaneously account for various stakeholders' weights; (2) supplying the sensitivity analysis of the weights; (3) validating multi-criteria scoring technique, particularly focusing on the ability of combing the DEA to identify the best and worst transferring transit nodes.

\section{Acknowledgements}

The research is supported by the National Natural Science Foundation of China (41271396). Data are collected under the National Undergraduate Training Program for Innovation and Entrepreneurship (201510486059).

\section{References}

[1] Chunqin Zhang, Zhicai Juan, Qingyu Luo, and Guangnian Xiao, 'Performance Evaluation of Public Transit Systems Using a Combined Evaluation Method', Transport Policy, 45 (2016), 156-67.

[2] Justin Jacobson, and Ann Forsyth, 'Seven American Tods: Good Practices for Urban Design in Transit-Oriented Development Projects', Journal of Transport and Land Use, 1 (2008).

[3] Joan E. Himann, David A. Cunningham, Peter A. Rechnitzer, and Donald H. Paterson, 'Age-Related Changes in Speed of Walking', Medicine and science in sports and exercise, 20 (1988), 161-66.

[4] Davide Malatesta, Luca Vismara, Francesco Menegoni, Manuela Galli, Marianna Romei, and Paolo Capodaglio, 'Mechanical External Work and Recovery at Preferred Walking Speed in Obese Subjects', Medicine+ Science in Sports+ Exercise, 41 (2009), 426.

[5] Catherine Holloway, and Nick Tyler, 'A Micro-Level Approach to Measuring the Accessibility of Footways for Wheelchair Users Using the Capability Model', Transportation Planning and Technology, 36 (2013), 636-49.

[6] Iderlina Mateo-Babiano, 'Pedestrian's Needs Matters: Examining Manila's Walking Environment', Transport Policy, 45 (2016), 107-15.

[7] Eric M. Delmelle, Shuping Li, and Alan T. Murray, 'Identifying Bus Stop Redundancy: A Gis-Based Spatial Optimization Approach', Computers, Environment and Urban Systems, 36 (2012), 445-55.

[8] Monsak Socharoentum, and Hassan A. Karimi, 'Multi-Modal Transportation with Multi-Criteria Walking (Mmt-Mcw): Personalized Route Recommender', Computers, Environment and Urban Systems, 55 (2016), 44-54.

[9] David S. Vale, 'Transit-Oriented Development, Integration of Land Use and Transport, and Pedestrian Accessibility: Combining Node-Place Model with Pedestrian Shed Ratio to Evaluate and Classify Station Areas in Lisbon', Journal of Transport Geography, 45 (2015), 70-80.

[10]Michael James Farrell, 'The Measurement of Productive Efficiency', Journal of the Royal 
Statistical Society. Series A (General) (1957), 253-90.

[11]Paul M. Hess, 'Measures of Connectivity [Streets: Old Paradigm, New Investment]', Places, 11 (1997). 